\title{
Static and Active Workstations for Improving Workplace Physical Activity and Sitting Time
}

\author{
K. Jason Crandall", Battogtokh Zagdsuren, Mark A. Schafer, T. Scott Lyons \\ School of Kinesiology, Recreation, and Sport, Western Kentucky University, USA
}

Copyright $(2016$ by authors, all rights reserved. Authors agree that this article remains permanently open access under the terms of the Creative Commons Attribution License 4.0 International License

\begin{abstract}
Purpose: There is growing evidence prolonged workplace sitting is associated with multiple health risks. Utilizing static and active workstations may help improve these health risks. The purpose of this investigation was to determine if providing access to a sit-stand workstation and a shared treadmill desk without a behavioural intervention could increase workplace physical activity and decrease sitting time. Methodology: Participants $(\mathrm{N}=15)$ were assigned to an experimental (sit-stand desk and shared treadmill desk; $n=8$ ) or control group $(\mathrm{n}=7)$ for seven weeks. No behavioural interventions were used to encourage participation. A physical activity questionnaire was administered at baseline and at the end of the study to assess workplace physical activity and sitting time. Participants wore an activity tracker (Fitbit One) to objectively assess daily footsteps and physically active hours. A mixed between-within subject analysis of variance was used to compare the groups $(p<.05)$. Findings: There were no statistically significant between-group differences in the dependent variables. Although just half of the experimental group used the shared treadmill desk, the mean number of footsteps taken (8897.25) was well above the recommended $>5000$ steps/day. Practical Implications: This is the first study to examine the use of both static and active workstations to increase workplace physical activity and reduce sitting time without a behavioural intervention. Value: Combining static and active workstations with the addition of a behavioural intervention to promote and motivate the participants may produce significant improvements in workplace physical activity and reductions in sitting time.
\end{abstract}

Keywords Prolonged Sitting, Sit-stand Desk, Treadmill Desk, Worksite Health Promotion, Physical Inactivity, Sedentary Time

\section{Purpose}

There is growing evidence prolonged sitting is associated with multiple health risks including musculoskeletal conditions, increased biomarkers of cardiovascular diseases, and increased mortality [1-2]. In fact, many researchers now consider excessive sitting to be comparable to smoking [3[. Indeed, high sitting populations ( $>10$ hours per day) were found to be at a $38 \%$ higher risk for myocardial infarction and $31 \%$ all-cause mortality versus low sitting populations $(<6$ hours per day) [4].

Excessive sitting can occur in three domains: sitting in the workplace, sitting while in transport and sitting in leisure time [3]. Multiple studies have sought to determine effective strategies for decreasing time spent sitting, or alternative methods for traditional chair sitting in order to reduce the deleterious effects of sedentary behaviour [5]. Because office-based employees spend most of their workday sitting, work-place interventions have the potential to significantly reduce overall sedentary behaviour [6]. Work-place behavioural interventions such as goal setting and education along with walking, climbing stairs, and structured exercise routines have been widely implemented with some success, but ergonomic interventions using static (sit-stand workstation) or active (treadmill desk) workstations may be a more feasible option [6-8].

Sit-stand workstations can be lowered and elevated to an appropriate height allowing employees to complete computer/desk-based tasks while alternating sitting and standing while performing job tasks (Figure 1). Alkhajah et al. [9] found sit-stand workstations significantly reduced office workers' sitting time during work and led to reduced sedentary time at home. Sit-stand workstations were also found to reduce upper back and neck pain, improve mood states, and reduce ambulatory blood pressure [10-11].

A treadmill desk is modified to allow employees to walk on a treadmill at a slow speed while continuing their job tasks (Figure 2). Koepp [12] replaced participants' traditional workstations with treadmill desks and found significant decreases in sedentary time, increases in daily physical activity, and significant weight loss in many of the participants. Ben-ner et al. [13] measured work performance using online performance surveys and found increases in physical activity and additional benefits of improved overall 
work performance and interactions with co-workers. Others showed treadmill desk walking can improve short-term increases in memory and attention along with health benefits [15].

Previous investigators have examined the use of behavioural interventions combined with ergonomic interventions, such as sit-stand workstations or treadmill desks, to reduce time sitting or increase physical activity. For most small to medium size businesses, however, placing a treadmill desk in every office-based employee's office along with the time required for goal-setting and education can be cost prohibitive. Perhaps an ergonomic intervention that uses both sit-stand workstations in each employee's office and provides a shared treadmill desk in a common area can successfully reduce workplace sitting time and workplace physical activity. In one of the few investigations to examine the effects of a shared treadmill desk, Schuna et al. [16] placed shared treadmill desks in a private company and found participants significantly increased daily footsteps (1622 steps/day) and light physical activity (1.6 minutes/hour). Therefore, the purpose of this investigation was to determine the effectiveness of using both sit-stand workstations and a shared treadmill desk without the addition of a behavioural intervention.

\section{Methodology}

\section{Participants}

A volunteer sample of faculty and staff in the School of Kinesiology, Recreation, and Sport at Western Kentucky University (WKU) were recruited using email notifications and word of mouth. Respondents were told the nature, risk, and potential benefits of the study. All participants completed a medical history questionnaire and the Physical Activity Readiness Questionnaire (PAR-Q) to determine exclusion criteria. The exclusion criteria for participation included a medical history that would prohibit use of the static and active workstations. The WKU Institutional Review Board (IRB \#645519-2) approved all procedures and written informed consent was obtained from each participant prior to beginning the investigation.

\section{Study Design}

Prior to the beginning of the seven week study, participants willing to have sit-stand workstations (Ergotron ${ }^{\circledR}$ WorkFit-S Dual Monitor Sit-Stand Desk with Work Surface) installed in their workspaces were assigned to the experimental group $(\mathrm{n}=8)$ (Figure 1). Participants were given the opportunity to choose a sit-stand workstation because installation requires modification of the work area. Participants could choose to either sit or stand throughout their workday because the sit-stand workstations were height adjustable. A brief introduction to the use of the sit-stand workstation was provided to each participant and each was told to choose to either sit or stand at their discretion over the course of the intervention. The researchers did not influence use of the sit-stand workstation in any way throughout the duration of the study. Log sheets were provided to record standing time and the number of sit-stand transitions. A research assistant collected the logs at the end of each week and a new log was provided. The workspace for the control group $(n=7)$ was not modified in any way.

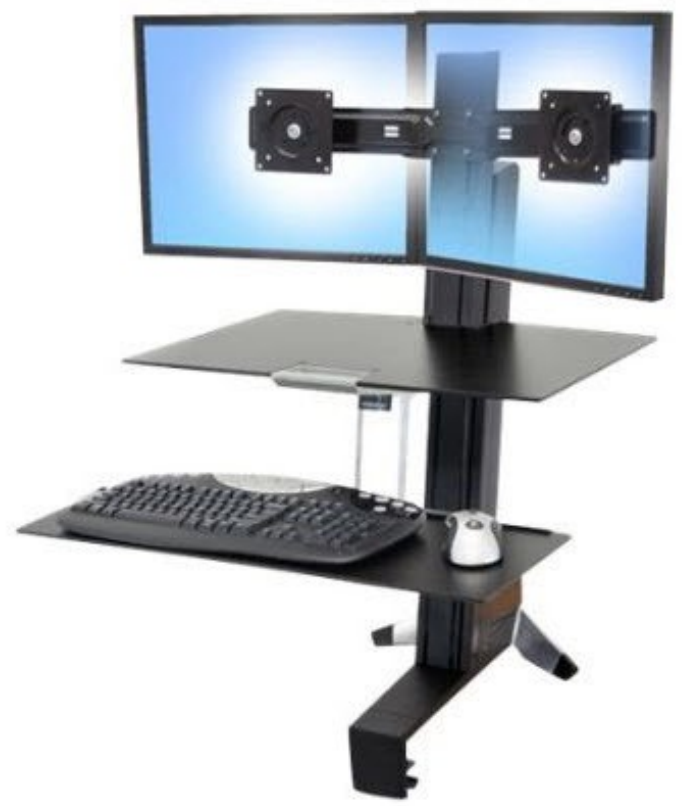

Figure 1. Ergotron ${ }^{\circledR}$ WorkFit-S Dual Monitor Sit-Stand Desk with Work Surface

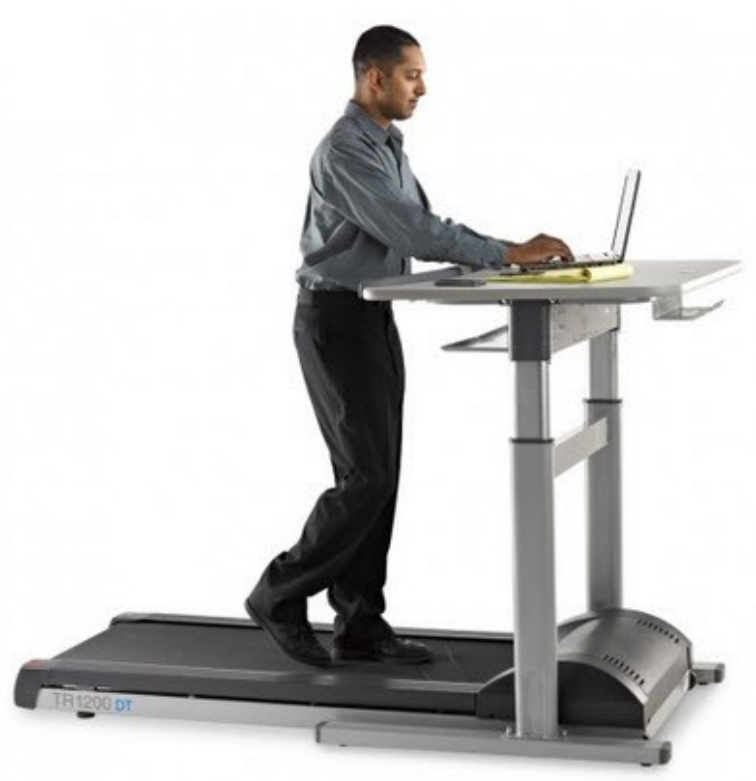

Figure 2. Lifespan TR1200-DT7

In addition to the sit-stand workstations, the experimental group was provided access to a shared electronically height-adjustable treadmill desk (Lifespan TR1200-DT7) located in a common room adjacent to the office space in the school of KRS (See Figure 2). Participants were given instructions regarding the use of the treadmill desk and access to the treadmill desk. They were not given any 
guidelines regarding how much to use the treadmill desk and to only use it at their discretion. The researchers did not influence use of the treadmill desk in any way throughout the duration of the study. Minutes, calories, and distance were recorded by the participant on a log sheet after each session. The log sheet was collected at the end of the study period.

\section{Outcome Measures}

The International Physical Activity Questionnaire (IPAQ) long form was administered at baseline and at the end of the study to workplace physical activity and workplace sitting time. The IPAQ is considered a valid and reliable self-report measure of the participant's last seven days of physical activity [16]. It measures four domains of physical activity (leisure time, domestic and gardening, work-related, and transport related). Within each domain walking, moderate-intensity, and vigorous-intensity activities can be assessed. For the purposes of this study, walking, moderate-intensity, and vigorous-intensity activities in the work-related domain are reported. The work-related domain calculations for these activities are:

1. Walking: MET-minutes/week at work $=3.3 \times$ walking minutes $\mathrm{x}$ walking days at work

2. Moderate-intensity: MET-minutes/week at work $=4.0 \mathrm{x}$ moderate-intensity activity minutes $x$ moderate-intensity days at work

3. Vigorous-intensity: MET-minutes/week at work $=8.0 \mathrm{x}$ vigorous-intensity activity minutes $\mathrm{x}$ vigorous-intensity days at work

The IPAQ also assesses workday sitting time. Total workday sitting hours per week were calculated using the equation: (weekday sitting minutes/60) x 5 weekdays.

To objectively assess total daily footsteps and physically active hours (light, moderately active, very active), participants wore a Fitbit One Wireless Activity Tracker (FIT; Fitbit Inc., San Francisco CA) in their pocket, on a belt, or on a bra throughout the study period. Intensity is calculated using Fitbit Inc. proprietary calculations. The FIT activity monitors are valid and reliable for physical activity tracking in healthy adults [17].

\section{Statistical Analysis}

Statistical analyses were obtained using the Statistical Package for the Social Sciences (SPSS, version 23.0, SPSS Inc, Chicago, IL). Independent samples t-tests and Pearson chi-square analyses were used to compare baseline participant characteristics. A mixed between-within subject ANOVA was used to examine group $\mathrm{x}$ time differences in IPAQ and FIT data. Alpha was set at $p<0.05$ and comparisons were two-tailed. The magnitude of any differences in the means was estimated using partial eta-squared $\left(\eta_{p}^{2}\right)$. Treadmill desk Minutes standing per workday, number of sit-stand transitions per workday, minutes walking, footsteps walking, and distance walked on the treadmill desk, footsteps were analysed to assess the impact of using a sit-stand desk and treadmill desk for the experimental group.

\section{Findings}

Sixteen $(N=16)$ participants were recruited for the study. One participant reported incomplete data and was not included in the analyses. The mean workday was $8.5 \pm 0.8$ and $9 \pm 0.6$ hours for the experimental group and control group, respectively. There were no significant differences in baseline participant characteristics between groups (Table 1).

Table 1. Baseline Participant Characteristics

\begin{tabular}{cccc}
\hline & $\begin{array}{c}\text { Control }(\mathrm{n}=7, \\
M(s d))\end{array}$ & $\begin{array}{c}\text { Experimental }(\mathrm{n}=8, M \\
(s d))\end{array}$ & p value \\
\hline Sex & $3(\mathrm{M}), 4(\mathrm{~F})$ & $5(\mathrm{M}), 3(\mathrm{~F})$ & .447 \\
Age (yrs) & $42.29(11.01)$ & $39.17(6.27)$ & .553 \\
Weight $(\mathrm{lbs})$ & $186.6(60.4)$ & $186.2(33)$ & .839 \\
Height (in) & $66(2.3)$ & $70.6(3.9)$ & .195 \\
BMI $\left(\mathrm{kg} / \mathrm{m}^{2}\right)$ & $29.7(7.6)$ & $26.6(0.6)$ & .572 \\
\hline
\end{tabular}

Note. $\mathrm{M}=$ Male, $\mathrm{F}=$ Female; ${ }^{*} \mathrm{p}<.05$

\section{IPAQ Results}

There were no significant group by time interactions for MET-minutes/week spent in walking, $F(1,12)=1.75$, p $=.211, \mathrm{y}_{\mathrm{p}}^{2}=.127$, MET-minutes $/$ week in moderate physical activity, $F(1,12)=.802, \mathrm{p}=.388, \mathrm{y}_{\mathrm{p}}{ }^{2}=.802$, MET-minutes/week in vigorous physical activity, or total workday hours spent sitting per week, $F(1,13)=.603, \mathrm{p}$ $=.452, \mathrm{y}_{\mathrm{p}}{ }^{2}=.044$. See Table 2 .

The main effect for time was not significant for walking, $F$ $(1,12)=.656, \mathrm{p}=.434$, moderate physical activity, $F(1,12)$ $=1.30, \mathrm{p}=.276, \mathrm{y}_{\mathrm{p}}^{2}=.098$, vigorous physical activity, $F(1$, $12)=2.21, \mathrm{p}=.163, \mathrm{y}_{\mathrm{p}}^{2}=.155$, or workday hours spent sitting, $F(1,13)=1.39, \mathrm{p}=.260, \mathrm{y}_{\mathrm{p}}^{2}=.096$.

Table 2. Group Comparisons for IPAQ Workplace Physical Activity and Minutes Sitting

\begin{tabular}{|c|c|c|c|c|}
\hline & \multicolumn{2}{|c|}{ Experimental $(M, s d)$} & \multicolumn{2}{|c|}{ Control $(M, s d)$} \\
\hline & Pre & Post & Pre & Post \\
\hline Walking $^{1}$ & $1291.06(1117.75)$ & $\begin{array}{l}1422.19 \\
(963.25)\end{array}$ & $\begin{array}{c}1013.21 \\
(1281.74)\end{array}$ & $\begin{array}{c}874.50 \\
(996.08) \\
\end{array}$ \\
\hline Moderate $^{1}$ Intensity & $1600.00(1624.21)$ & $\begin{array}{c}1896.25 \\
(1981.05)\end{array}$ & $\begin{array}{c}1271.43 \\
(1017.70 \\
\end{array}$ & $\begin{array}{c}1945.71 \\
(1898.52) \\
\end{array}$ \\
\hline Vigorous ${ }^{1}$ Intensity & $880.00(877.40)$ & $\begin{array}{c}821.88 \\
(906) \\
\end{array}$ & $\begin{array}{c}1337.14 \\
(1412.11)\end{array}$ & $\begin{array}{c}1068.57 \\
(1538.61) \\
\end{array}$ \\
\hline Workday Sitting (hrs/wk) & $7.25(2.76)$ & $4.50(2.78)$ & $6.0(2.52)$ & $6.5(2.78)$ \\
\hline
\end{tabular}

Note. ${ }^{*} \mathrm{p}<.05 ;$ MET-minutes/week 
Table 3. Group Comparisons for FIT Footsteps and Physical Activity

\begin{tabular}{|c|c|c|c|c|}
\hline & \multicolumn{2}{|c|}{ Experimental } & \multicolumn{2}{c|}{ Control } \\
\hline & Pre $(M, \mathrm{sd})$ & $\operatorname{Post}(M, \mathrm{sd})$ & $\operatorname{Pre}(M, \mathrm{sd})$ & Post $(M, \mathrm{sd})$ \\
\hline Daily footsteps & $\begin{array}{c}9416.86 \\
(3696.41)\end{array}$ & $\begin{array}{c}6926.22^{*} \\
(1807.62)\end{array}$ & $\begin{array}{c}7399.66 \\
(2499.83)\end{array}$ & $6118.63^{*}(1320.27)$ \\
\hline Light (hours/wk) & $2.78(1.05)$ & $2.91(1.16)$ & $3.05(0.48)$ & $2.51(0.71)$ \\
\hline Moderate (hours/wk) & $1.19(0.63)$ & $1.16(0.28)$ & $1.30(0.83)$ & $1.03(0.45)$ \\
\hline Very Active (hours/wk) & $0.61(0.47)$ & $0.26(0.17)$ & $0.32(0.20)$ & $0.34(0.38)$ \\
\hline
\end{tabular}

Note. $* \mathrm{p}<.05$

Table 4. Sit-Stand Workstation and Treadmill Desk Results

\begin{tabular}{|c|c|}
\hline Variables & Experimental $(M, s d)$ \\
\hline Minutes standing per workday & $84.6(45.56)$ \\
\hline Number of sit-stand transitions per workday & $1.91(0.59)$ \\
\hline Minutes walking on treadmill desk* & $108.5(88.02)$ \\
\hline Footsteps walking on treadmill desk* & $8897.25(7637.14)$ \\
\hline Distance walked on treadmill desk (miles)* & $3.45(2.88)$ \\
\hline
\end{tabular}

Note. *Four of the eight experimental group participants used the treadmill desk

\section{FIT Results}

There were no significant group by time interactions for participants' daily footsteps, $F(1,12)=1.18, \mathrm{p}=.298, \mathrm{y}_{\mathrm{p}}{ }^{2}$ $=.090$, light physical activity, $F(1,12)=1.75, \mathrm{p}=.211, \mathrm{y}_{\mathrm{p}}{ }^{2}$ $=.127$, moderate physical activity, $F(1,12)=.802, \mathrm{p}=.388$, $\mathrm{\eta}_{\mathrm{p}}{ }^{2}=.063$, or vigorous physical activity, $F(1,12)=3.09, \mathrm{p}$ $=.104, \mathrm{y}_{\mathrm{p}}{ }^{2}=.205$.

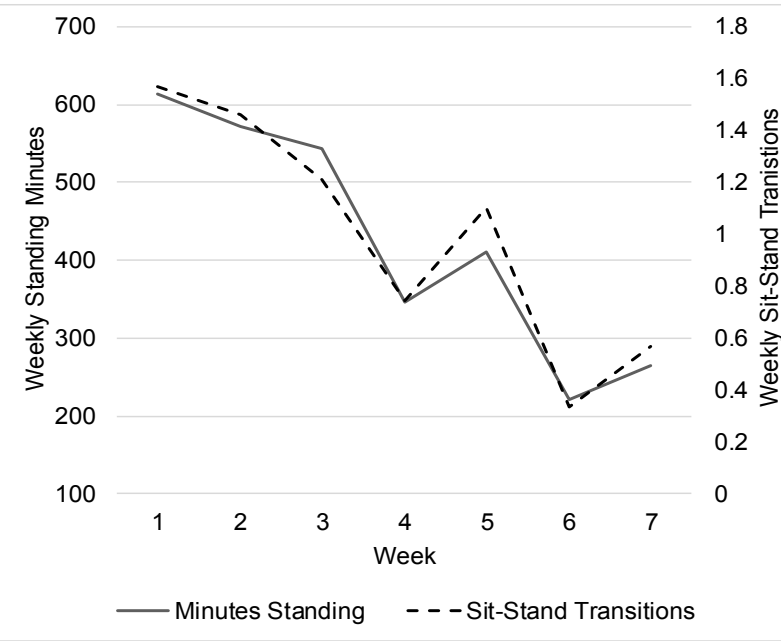

Figure 3. Comparison of Minutes Standing and Sit-Stand Transitions

There were no significant main effects for light physical activity, $F(1,12)=.656, \mathrm{p}=.434, \mathrm{n}_{\mathrm{p}}{ }^{2}=.052$, moderately physically active, $F(1,12)=1.30, \mathrm{p}=.276, \mathrm{p}_{\mathrm{p}}{ }^{2}=.098$, or very physically active, $F(1,12)=.265, \mathrm{p}=.129, \mathrm{y}_{\mathrm{p}}{ }^{2}=.181$. There was a significant main effect for time for participants' daily footsteps, $\mathrm{F}(1,12)=11.49, \mathrm{p}=.005, \mathrm{y}_{\mathrm{p}}{ }^{2}=.489$ where both the experimental and control groups' daily footsteps significantly decreased across the study period. See Table 3.

The experimental group stood an average of 84.6 minutes per workday. Half of the experimental group participants used the treadmill desk at least once during the study period. See Table 4. Total workday hours standing and number of sit-stand transitions decreased each week across the intervention period.

\section{Discussion}

The purpose of this investigation was to determine the effectiveness of using both sit-stand workstations and a shared treadmill desk without the addition of a behavioural intervention. There were no significant differences in workplace physical activity, total daily footsteps, or workplace sitting time at the end of our study. Although we did not find significant differences there were several interesting findings from this investigation.

First, we found that when the shared treadmill desk is utilized, it can have a positive impact on daily workplace footsteps. Although just half of the experimental participants used the shared treadmill desk, the mean number of footsteps (8897.25) taken was well above the $>5000$ steps/day recommended as a step-defined sedentary lifestyle index for adults [6].

Second, we chose to study the effects of a shared treadmill desk because most businesses and organizations will not have the financial resources or sufficient infrastructure to place treadmill desks in all employees' offices. However, because the treadmill desk was placed in a conference room, there were instances when participants could not use it due to scheduling conflicts. Also, because the treadmill desk was not centrally located to their offices some participants forgot it was available.

Previous researchers have successfully increased workplace physical activity by placing and promoting treadmill desks in individual employees' offices [12-13, 16], but it is evident from the current investigation that a treadmill 
desk in the common area limits the ability for some to use it.

Another interesting finding was weekly sit-stand workstation use and number of transitions gradually declined across the investigation. This could possibly due to the reduced novelty of the equipment. Some participants also reported sore knees and backs as well as fatigue from exercise sessions. These problems limited the participants' willingness to stand throughout the day. Dutta et al. [19] reported similar fatigue after their investigation.

A positive outcome was that even with the weekly decline in sit-stand workstation use, the experimental group averaged 84.6 minutes of standing per workday. Despite evidence suggesting this level of energy expenditure is unlikely to result in meaningful body weight loss, others have shown simply breaking up prolonged sitting time with periods of standing or light physical activity can improve measures of health including endothelial cell function, ambulatory blood pressure, and altered expression of genes involved in carbohydrate metabolism [19-22].

Finally, previous researchers have successfully used a multicomponent approach that combined static or active workstations with a behavioural intervention that included education workshops, reminder emails, or health coaching [23]. Because behavioural interventions can be resource intensive, we introduced the sit-stand workstations and shared treadmill desk without any behavioural intervention to determine if simply changing the work environment could positively affect physical activity and sitting time. Although no significant differences were found, the experimental group did increase self-reported minutes walking and decreased workday sitting hrs/wk. Our results suggest a multicomponent intervention that includes a behavioural component may be necessary to realize statistically significant improvements in physical activity and siting time.

There were some potential limitations to this investigation. First, there were positive trends in the outcomes for this investigation, yet the small sample size limited our ability to detect statistically significant differences. Second, a more objective measure to detect time sitting and standing may be needed for future investigations since participants may have forgotten to record their standing time. Finally, because we used a non-random sample of physically active faculty members from the WKU School of Kinesiology, Recreation, and Sport, these data may not be generalizable to the employee population at WKU.

\section{Value}

To our knowledge, this is the first investigation to examine the effectiveness of using both sit-stand workstations and a shared treadmill desk without the addition of a behavioural intervention. Although we did not find significant improvements in this investigation, we believe combining sit-stand workstations with a shared treadmill desk has the potential for increasing workplace physical activity and reducing sitting time, however, future investigations will need to address employees' barriers to their use and combine both ergonomic and behavioural interventions.

\section{Acknowledgements}

The authors wish to thank Wade Pinkard and Tony Glisson with Western Kentucky University for their financial support of this project.

\section{Funding Sources}

This study was supported by funding from the Western Kentucky University Health Services and Human Resources Department, School of Kinesiology, Recreation, and Sport, Department of Environmental Health and Safety, Workers' Compensation Program, Employee Wellness Program, and the Division of Finance and Administration.

\section{Author Contributions}

$\mathrm{N} / \mathrm{A}$

\section{Disclosures and Ethics}

N/A

\section{REFERENCES}

[1] Healy, G. N., Matthews, C. E., Dunstan, D. W., Winkler, E. A. \& Owen N. 2011. Sedentary time and cardio-metabolic biomarkers in US adults: NHANES 2003-06. Eur Heart J, 32, 590-7.

[2] Gibbs, B. B., Hergenroeder, A. L., Katzmarzyk, P. T., Lee, I. M. \& Jakicic, J. M. 2014. Definition, Measurement, and Health Risks Associated with Sedentary Behavior. Med Sci Sports Exerc.

[3] Gerstacker, D. (2014). Sitting is the new smoking-7 ways a sedentary lifestyle is killing you. Retrieved from $\mathrm{http}: / /$ www.theactivetimes.com/sitting-new-smoking-7-ways -sedentary-lifestyle-killing-you?utm_source=huffington $\% 2 \mathrm{~B}$ post\&utm_medium=partner\&utm_campaign $=$ sitting

[4] Bjork Petersen, C., Bauman, A., Gronbaek, M., Wulff Helge, J., Thygesen, L. C. \& Tolstrup, J. S. 2014. Total sitting time and risk of myocardial infarction, coronary heart disease and all-cause mortality in a prospective cohort of Danish adults. Int J Behav Nutr Phys Act, 11, 13.

[5] Neuhaus, M., Healy, G. N., Dunstan, D. W., Owen, N. \& Eakin, E. G. 2014a. Workplace sitting and height-adjustable workstations: a randomized controlled trial. Am J Prev Med, 46, 30-40.

[6] Tudpr-Locke, C., Schuna, J. M., JR., Frensham, L. J. \& Proenca, M. 2014. Changing the way we work: elevating 
energy expenditure with workstation alternatives. Int $J$ Obes (Lond), 38, 755-65.

[7] Edmunds, S., Hurst, L. \& Harvey, K. 2013. Physical activity barriers in the workplace. International Journal of Workplace Health Management, 6, 227-240.

[8] Tew, G. A., Posso, M. C., Arundel, C. E. \& McDaid, C. M. 2015. Systematic review: height-adjustable workstations to reduce sedentary behaviour in office-based workers. Oсcup Med (Lond), 65, 357-66.

[9] Alkhajah, T. A., Reevers, M. M., Eakin, E. G., Winkler, E. A., Owen, N. \& Healy, G. N. 2012. Sit-stand workstations: a pilot intervention to reduce office sitting time. Am J Prev Med, 43, 298-303.

[10] Pronk, N. P. 2009. Physical activity promotion in business and industry: evidence, context, and recommendations for a national plan. $J$ Phys Act Health, 6 Suppl 2, S220-35.

[11] Zeigler, Z. S., Mullane, S., Crespo, N. C., Buman, M. P. \& Gaesser, G. A. 2015. Effects of Standing and Light-Intensity Activity on Ambulatory Blood Pressure. Med Sci Sports Exerc. 48(2):175-81

[12] Koepp, G. A., Manohar, C. U., McCrady-Spitzer, S. K., Ben-Ner, A., Hamann, D. J., Runge, C. F., \& Levine, J. A. (2013). Treadmill desks: A 1-year prospective trial. Obesity (Silver Spring), 21(4), 705-711. doi:10.1002/oby.20121

[13] Ben-Ner, A., Hamann, D. J., Koepp, G., Manohar, C. U., \& Levine, J. (2014). Treadmill workstations: the effects of walking while working on physical activity and work performance. PLoS One, 9(2), e88620. doi:10.1371/journal.p one. 0088620

[14] Labonte-LeMoyne, E., Santhanam, R., Leger, P. M., Courtemanche, F., Fredette, M., \& Senecal, S. (2015). The delayed effect of treadmill desk usage on recall and attention. Computers in Human Behavior, 46, 1-5. doi:10.1016/j.chb.2 014.12 .054

[15] Schuna, J. M., Jr., Swift, D. L., Hendrick, C. A., Duet, M. T., Johnson, W. D., Martin, C. K., . . . Tudor-Locke, C. (2014). Evaluation of a workplace treadmill desk intervention: a randomized controlled trial. J Occup Environ Med, 56(12), 1266-1276. doi:10.1097/JOM.0000000000000336

[16] Craig, C. L., Marshall, A. L., Sjostrom, M., Bauman, A. E., Booth, M. L., Ainsworth, B. E., ... Oja, P. (2003). International physical activity questionnaire: 12-country reliability and validity. Med Sci Sports Exerc, 35(8), 1381-1395. doi:10.1249/01.MSS.0000078924.61453.FB

[17] Takacs, J., Pollock, C. L., Guenther, J. R., Bahar, M., Napier, C., \& Hunt, M. A. (2014). Validation of the Fitbit One activity monitor device during treadmill walking. J Sci Med Sport, 17(5), 496-500. doi:10.1016/j.jsams.2013.10.241

[18] Dutta, N., Koepp, G. A., Stovitz, S. D., Levine, J. A., \& Pereira, M. A. (2014). Using sit-stand workstations to decrease sedentary time in office workers: a randomized crossover trial. Int J Environ Res Public Health, 11(7), 6653-6665. doi:10.3390/ijerph110706653

[19] Latouche, C., Jowett, J. B., Carey, A. L., Bertovic, D. A., Owen, N., Dunstan, D. W., \& Kingwell, B. A. (2013). Effects of breaking up prolonged sitting on skeletal muscle gene expression. J Appl Physiol (1985), 114(4), 453-460. doi:10.1152/japplphysiol.00978.2012

[20] Thosar, S. S., Bielko, S. L., Mather, K. J., Johnston, J. D., \& Wallace, J. P. (2015). Effect of Prolonged Sitting and Breaks in Sitting Time on Endothelial Function. Medicine \& Science in Sports \& Exercise, 47(4), 843-849. doi: 10.1249/mss.0000 000000000479

[21] Tudor-Locke, C., Schuna, J. M., Jr., Frensham, L. J., \& Proenca, M. (2014). Changing the way we work: elevating energy expenditure with workstation alternatives. Int $J$ Obes (Lond), 38(6), 755-765. doi:10.1038/ijo.2013.223

[22] Zeigler, Z. S., Mullane, S., Crespo, N. C., Buman, M. P., \& Gaesser, G. A. (2015). Effects of Standing and Light-Intensity Activity on Ambulatory Blood Pressure. Med Sci Sports Exerc. doi:10.1249/MSS.0000000000000754

[23] Neuhaus, M., Healy, G. N., Dunstan, D. W., Owen, N., \& Eakin, E. G. (2014). Workplace sitting and height-adjustable workstations: a randomized controlled trial. Am J Prev Med, 46(1), 30-40. doi:10.1016/j.amepre.2013.09.009 